\title{
Linkage Disequilibrium Inflates Type I Error Rates in Multipoint Linkage Analysis when Parental Genotypes Are Missing
}

\author{
Abee L. Boyles William K. Scott Eden R. Martin Silke Schmidt \\ Yi-Ju Li Allison Ashley-Koch Meredyth P. Bass Michael Schmidt \\ Margaret A. Pericak-Vance Marcy C. Speer Elizabeth R. Hauser \\ Center for Human Genetics, Duke University Medical Center, Durham, N.C., USA
}

\section{Key Words}

Linkage disequilibrium - Measures of linkage

disequilibrium - Linkage analysis - False positive rate

Parameter misspecification

\begin{abstract}
Objectives: Describe the inflation in nonparametric multipoint LOD scores due to inter-marker linkage disequilibrium (LD) across many markers with varied allele frequencies. Method: Using simulated two-generation families with and without parents, we conducted nonparametric multipoint linkage analysis with 2 to 10 markers with minor allele frequencies (MAF) of 0.5 and 0.1 . Results: Misspecification of population haplotype frequencies by assuming linkage equilibrium caused inflated multipoint LOD scores due to inter-marker LD when parental genotypes were not included. Inflation increased as more markers in LD were included and decreased as markers in equilibrium were added. When marker allele frequencies were unequal, the $r^{2}$ measure of LD was a better predictor of inflation than $\mathrm{D}^{\prime}$. Conclusion: This observation strongly supports the evaluation of LD in multipoint linkage analyses, and further sug-
\end{abstract}

gests that unaccounted for LD may be suspected when two-point and multipoint linkage analyses show a marked disparity in regions with elevated $r^{2}$ measures of LD. Given the increasing popularity of high-density genomewide SNP screens, inter-marker LD should be a concern in future linkage studies.

Copyright $\odot 2005$ S. Karger AG, Basel

\section{Introduction}

High-density SNP mapping for analysis of a candidate region has become a common and powerful approach for localizing disease genes [1]. Dense maps of SNPs are more likely to contain markers with strong linkage disequilibrium (LD) than sparse microsatellite maps. Knowledge of these patterns of LD in a candidate gene region is necessary, and may require new methods of linkage analysis [2]. Most current linkage analysis programs require the simplifying assumptions of Hardy-Weinberg equilibrium at all loci and linkage equilibrium between alleles at different loci.

Haplotype frequencies can be incorrectly inferred when inter-marker LD is unaccounted for leading to in-

\section{KARGER \\ Fax +41613061234 E-Mail karger@karger.ch} www.karger.com
(C) 2005 S. Karger AG, Basel 0001-5652/05/0594-0220\$22.00/0

Accessible online at:

www.karger.com/hhe
Elizabeth R. Hauser, PhD

Duke University Medical Center

Box 3445

Durham, NC 27710 (USA)

Tel. +1 919684 2063, Fax +1 919684 2275, E-Mail Elizabeth.Hauser@duke.edu 
flated multipoint LOD scores when parental genotypes are missing. Here, the haplotype frequencies are analogous to allele frequencies for a multiallelic marker, and the detrimental effects of allele frequency misspecification on linkage analysis have been well documented [3, 4]. Particularly when a disease locus is between closely spaced markers, multipoint linkage analysis is not robust to parameter misspecification [5]. When parental genotypes are available, pre-specified allele frequencies do not contribute to the LOD score calculation and the results are immune to the effects of inter-marker LD.

Extensive variation of linkage disequilibrium in the genome makes direct study of its effect on analysis difficult. Recently published work by Huang et al. highlighted the potential for LD-biased LOD scores in sibling pair studies [6]. We independently replicated their findings of inflated multipoint LOD scores due to inter-marker LD for affected sibling pairs without parental genotypes. In this work we report an expanded study of this phenomenon including nonparametric methods and up to 10 markers at varied levels of LD. We also compared two common measures of $\mathrm{LD}, \mathrm{D}^{\prime}$ and $\mathrm{r}^{2}$ to determine which is superior for examination of the effect of LD on LOD score inflation. By including SNPs with common and rare minor allele frequencies we observed LOD scores when $\mathrm{D}^{\prime}$ was high and $\mathrm{r}^{2}$ was low.

\section{Methods}

Utilizing the SIMLA (SIMulation of Linkage and Association) software package [7], 10,000 replicates of 200 affected sibling pair families each were simulated. Two family structures with two affected siblings were included: complete nuclear families (parents and offspring), often studied in early onset disorders such as neural tube defects or autism, and affected sibling pairs (no parents) more typical of late onset disorders, such as Alzheimer or Parkinson disease. At no time was a disease locus included in any simulation such that all elevated LOD scores are spurious. Table 1 summarizes the parameters varied in these simulation studies.

SIMLA allows for the simulation of LD between markers and/or disease loci by specifying the frequencies of haplotypes on chromosomes. Inter-marker LD between two or four markers with equal allele frequencies $0.0002 \mathrm{c} M$ apart was specified by adjusting the haplotype frequencies to reflect levels of LD from 0 to 1 as measured by $\mathrm{D}^{\prime}$. Two, four, or six additional markers in linkage equilibrium with all markers were then added $5 \mathrm{c} M$ on either side of the original markers (fig. 1). Nonparametric multipoint analysis of up to ten markers was performed using SIBLINK [8], while initial parametric analyses were performed with VITESSE.

With the underlying assumption of linkage equilibrium twopoint and multipoint LOD scores rely on the generating allele frequencies to infer parental genotypes. Even though the allele frequencies are correctly specified, LD between the markers leads to
Table 1. Simulated model parameters

\begin{tabular}{llll}
\hline Model & $\begin{array}{l}\text { Markers } \\
\text { in LD }\end{array}$ & $\begin{array}{l}\text { Flanking } \\
\text { markers }\end{array}$ \\
\hline Additive & 2 & 0 & $\begin{array}{l}\text { (a) Common } \times \text { Rare } \\
\text { and Rare } \times \text { Rare (fig. 4) }\end{array}$ \\
Additive & 4 & 0 & \\
Additive & 2 & 2 & \\
Additive & 4 & 2 & \\
Additive & 2 & 4 & \\
Additive & 4 & 4 & \\
Additive & 2 & 6 & \\
Additive & 4 & 6 &
\end{tabular}

Multipoint and two-point analyses were performed for the following scenarios with and without parental genotypes All simulations were conducted at 6 levels of $\mathrm{LD}\left(\mathrm{D}^{\prime}=0,0.2,0.4,0.6,0.8\right.$, 1.0). All scenarios were run for markers with equal allele frequencies, (a) except the two cases noted where Common $\times$ Rare and Rare $\times$ Rare combinations of markers in LD were also analyzed without parental genotypes.

misspecification of the haplotype frequencies for multipoint analysis as illustrated in table 2. Analogous to the case of misspecified allele frequencies, as the misspecified haplotype frequencies get smaller the assumed probability that the haplotype is shared IBD increases, as does the false positive rate. For example when $\mathrm{D}^{\prime}=1$ the true haplotype frequency is 0.5 for the two observed haplotypes but the assumed frequency is 0.0625 , notably smaller. Calculating a LOD score with haplotype frequency of 0.0625 instead of 0.5 would inflate the LOD score (and type I error) because the assumed haplotype frequency of 0.0625 results in a higher probability that observed allele sharing IBS is due to sharing alleles IBD than would be obtained from using the true haplotype frequency of 0.5 .

To examine the influence of misspecified haplotype frequencies (and the effect of different numbers of markers in different degrees of LD on that haplotype misspecification) on LOD scores, two allele frequency scenarios were simulated; first, markers with equally frequent alleles and second, markers with minor allele frequencies of 0.5 and 0.1 . Two markers with common alleles, two with rare alleles, or one of each type were analyzed for 2 markers in LD. By varying the allele frequencies, we reduced the $\mathrm{r}^{2}$ value while holding the $\mathrm{D}^{\prime}$ value constant (fig. 2).

For evaluating the false positive rate, data were generated without linkage to a disease locus. LOD score thresholds of 1, 2, or 3 were considered as cutoffs for significant linkage. No disease locus was simulated thus all LOD scores over the threshold indicate a false positive result. Results were compared for multipoint analysis with two to ten markers with and without parental genotypes and with varying levels of $L D$. 


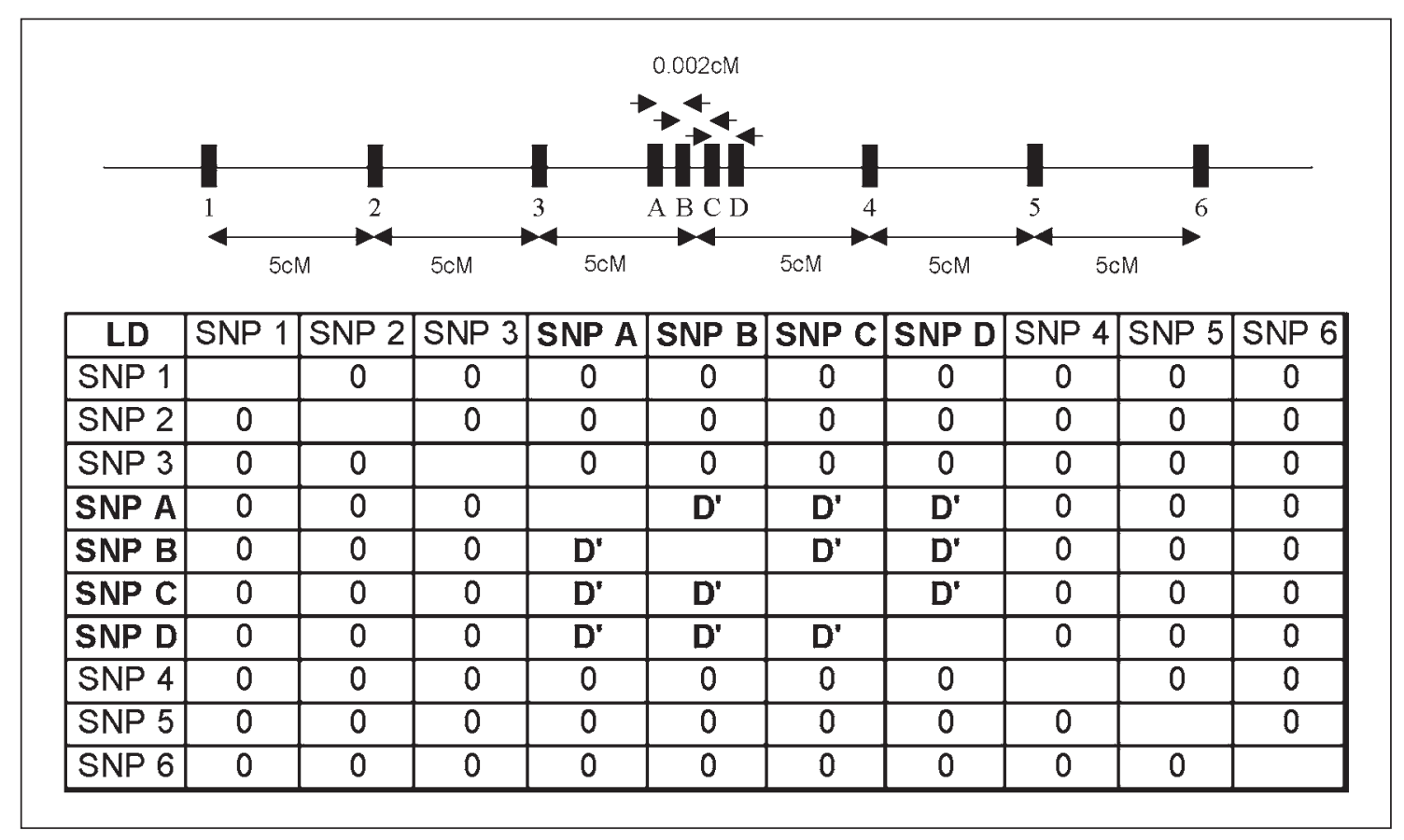

Fig. 1. Schematic of the ten markers and the simulated pair-wise LD between them. The addition of markers in equilibrium decreased, but did not eliminate the inflated false positive rate when parental genotypes were missing. Multipoints over multiple sets of markers in high LD further inflated LOD scores.

Table 2. Deviation between the proportion of haplotypes expected under linkage equilibrium and those observed with LD between the four biallelic markers as outlined in figure 1

\begin{tabular}{|c|c|c|c|c|c|c|c|}
\hline \multirow[t]{2}{*}{ Haplotype } & \multirow{2}{*}{$\begin{array}{l}\text { Expected } \\
\text { proportions }\end{array}$} & \multicolumn{6}{|c|}{ Observed proportions } \\
\hline & & $\mathrm{D}^{\prime}=0.0$ & $\mathrm{D}^{\prime}=0.2$ & $\mathrm{D}^{\prime}=0.4$ & $\mathrm{D}^{\prime}=0.6$ & $\mathrm{D}^{\prime}=0.8$ & $\mathrm{D}^{\prime}=1.0$ \\
\hline $\mathrm{A}_{1} \mathrm{~B}_{1} \mathrm{C}_{1} \mathrm{D}_{1}$ & 0.0625 & 0.0625 & 0.15 & 0.2375 & 0.325 & 0.4125 & 0.5 \\
\hline $\mathrm{A}_{1} \mathrm{~B}_{1} \mathrm{C}_{1} \mathrm{D}_{2}$ & 0.0625 & 0.0625 & 0.05 & 0.0375 & 0.025 & 0.0125 & 0 \\
\hline $\mathrm{A}_{1} \mathrm{~B}_{1} \mathrm{C}_{2} \mathrm{D}_{1}$ & 0.0625 & 0.0625 & 0.05 & 0.0375 & 0.025 & 0.0125 & 0 \\
\hline$A_{1} B_{1} C_{2} D_{2}$ & 0.0625 & 0.0625 & 0.05 & 0.0375 & 0.025 & 0.0125 & 0 \\
\hline $\mathrm{A}_{1} \mathrm{~B}_{2} \mathrm{C}_{1} \mathrm{D}_{1}$ & 0.0625 & 0.0625 & 0.05 & 0.0375 & 0.025 & 0.0125 & 0 \\
\hline $\mathrm{A}_{1} \mathrm{~B}_{2} \mathrm{C}_{1} \mathrm{D}_{2}$ & 0.0625 & 0.0625 & 0.05 & 0.0375 & 0.025 & 0.0125 & 0 \\
\hline $\mathrm{A}_{1} \mathrm{~B}_{2} \mathrm{C}_{2} \mathrm{D}_{1}$ & 0.0625 & 0.0625 & 0.05 & 0.0375 & 0.025 & 0.0125 & 0 \\
\hline $\mathrm{A}_{1} \mathrm{~B}_{2} \mathrm{C}_{2} \mathrm{D}_{2}$ & 0.0625 & 0.0625 & 0.05 & 0.0375 & 0.025 & 0.0125 & 0 \\
\hline $\mathrm{A}_{2} \mathrm{~B}_{1} \mathrm{C}_{1} \mathrm{D}_{1}$ & 0.0625 & 0.0625 & 0.05 & 0.0375 & 0.025 & 0.0125 & 0 \\
\hline $\mathrm{A}_{2} \mathrm{~B}_{1} \mathrm{C}_{1} \mathrm{D}_{2}$ & 0.0625 & 0.0625 & 0.05 & 0.0375 & 0.025 & 0.0125 & 0 \\
\hline $\mathrm{A}_{2} \mathrm{~B}_{1} \mathrm{C}_{2} \mathrm{D}_{1}$ & 0.0625 & 0.0625 & 0.05 & 0.0375 & 0.025 & 0.0125 & 0 \\
\hline $\mathrm{A}_{2} \mathrm{~B}_{1} \mathrm{C}_{2} \mathrm{D}_{2}$ & 0.0625 & 0.0625 & 0.05 & 0.0375 & 0.025 & 0.0125 & 0 \\
\hline $\mathrm{A}_{2} \mathrm{~B}_{2} \mathrm{C}_{1} \mathrm{D}_{1}$ & 0.0625 & 0.0625 & 0.05 & 0.0375 & 0.025 & 0.0125 & 0 \\
\hline $\mathrm{A}_{2} \mathrm{~B}_{2} \mathrm{C}_{1} \mathrm{D}_{2}$ & 0.0625 & 0.0625 & 0.05 & 0.0375 & 0.025 & 0.0125 & 0 \\
\hline $\mathrm{A}_{2} \mathrm{~B}_{2} \mathrm{C}_{2} \mathrm{D}_{1}$ & 0.0625 & 0.0625 & 0.05 & 0.0375 & 0.025 & 0.0125 & 0 \\
\hline $\mathrm{A}_{2} \mathrm{~B}_{2} \mathrm{C}_{2} \mathrm{D}_{2}$ & 0.0625 & 0.0625 & 0.15 & 0.2375 & 0.325 & 0.4125 & 0.5 \\
\hline
\end{tabular}


Fig. 2. The relationship between $D^{\prime}$ and $r^{2}$ when both allele frequencies are common $(\mathrm{MAF}=0.5)$, one is common and one is rare $(\mathrm{MAF}=0.1)$, or both are rare. When allele frequencies are disparate, there is a strong discrepancy between the $\mathrm{D}^{\prime}$ and $\mathrm{r}^{2}$ measures.

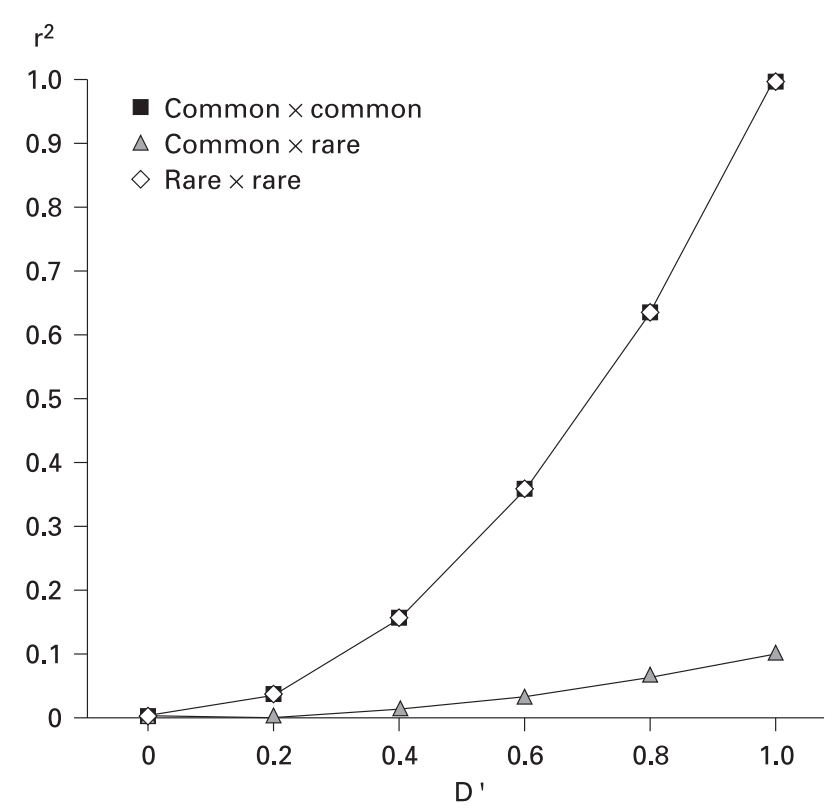

(fig. 3). When 4 markers are in $\mathrm{LD}\left(\mathrm{D}^{\prime}=1\right)$, the proportion of scores over 1 is $60 \%$ without flanking markers, $35 \%$ for two, $4.4 \%$ for four, and $5.4 \%$ for six flanking markers. As expected, no inflation was seen in two-point scores or when parental genotypes were included (data not shown).

\section{$r^{2}$ Is a Better Predictor of Inflation when Allele}

Frequencies Are Disparate

Introducing markers with a MAF of 0.1 creates a discrepancy between two common measures of LD, $\mathrm{D}^{\prime}$ and $r^{2}$. $D^{\prime}$ is scaled from -1 to 1 by the observed marker allele frequencies such that a $\mathrm{D}^{\prime}$ of 0 indicates no $\mathrm{LD}$ and a $\mathrm{D}^{\prime}$ of -1 or 1 indicates the maximum possible LD given those allele frequencies. Another measure, $\mathrm{r}^{2}$, is a squared correlation coefficient and denotes the ability of alleles at one marker to predict alleles at the second marker. When two markers have equal allele frequencies $r^{2}$ will be equal to $\left(D^{\prime}\right)^{2}$, but when the allele frequencies at the two markers are different the $r^{2}$ will not maximize to 1 (fig. 2).

In the one common marker and one rare marker case, $\mathrm{r}^{2}$ maximizes to 0.11 and no inflation of LOD scores is observed in the scenario of two markers in LD (fig. 4). 


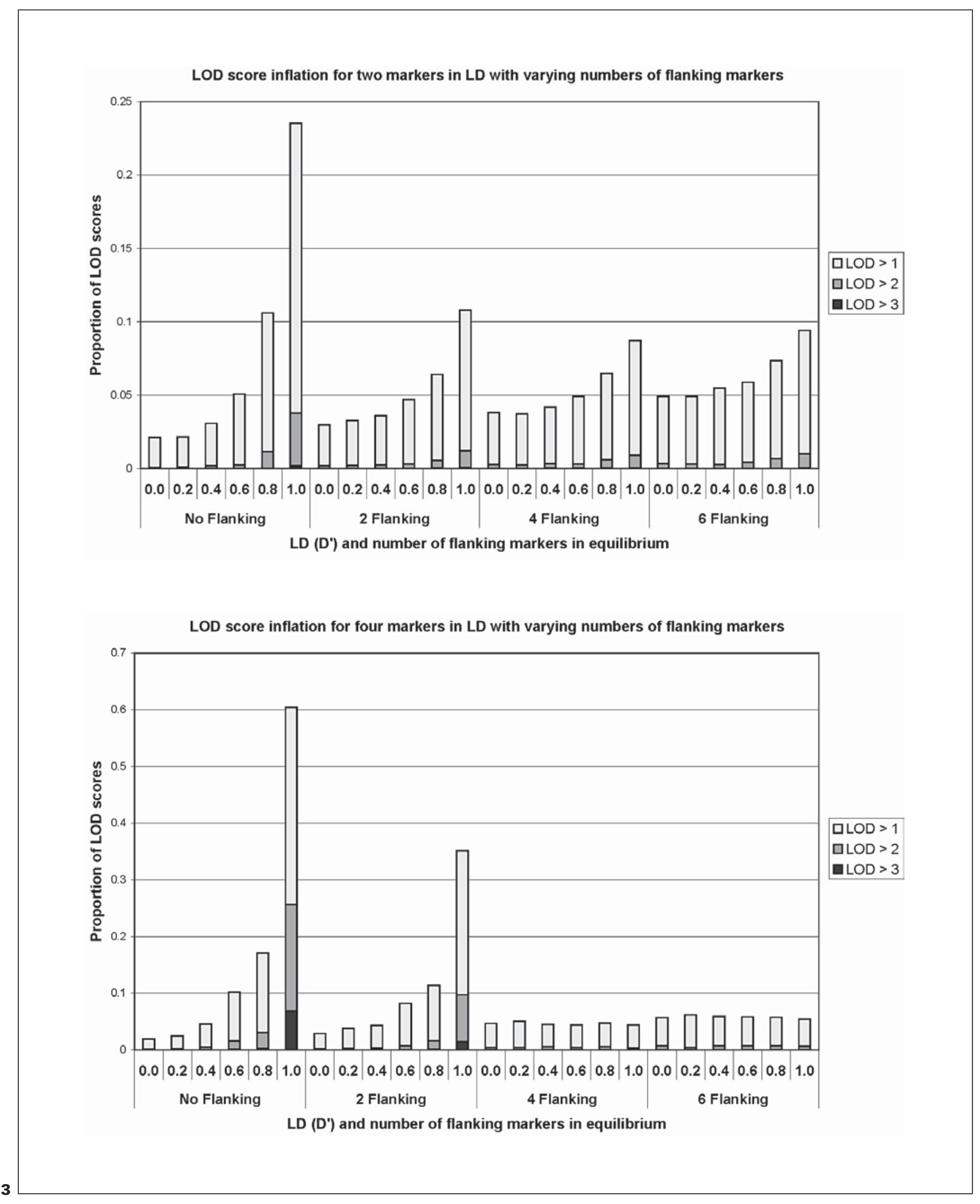




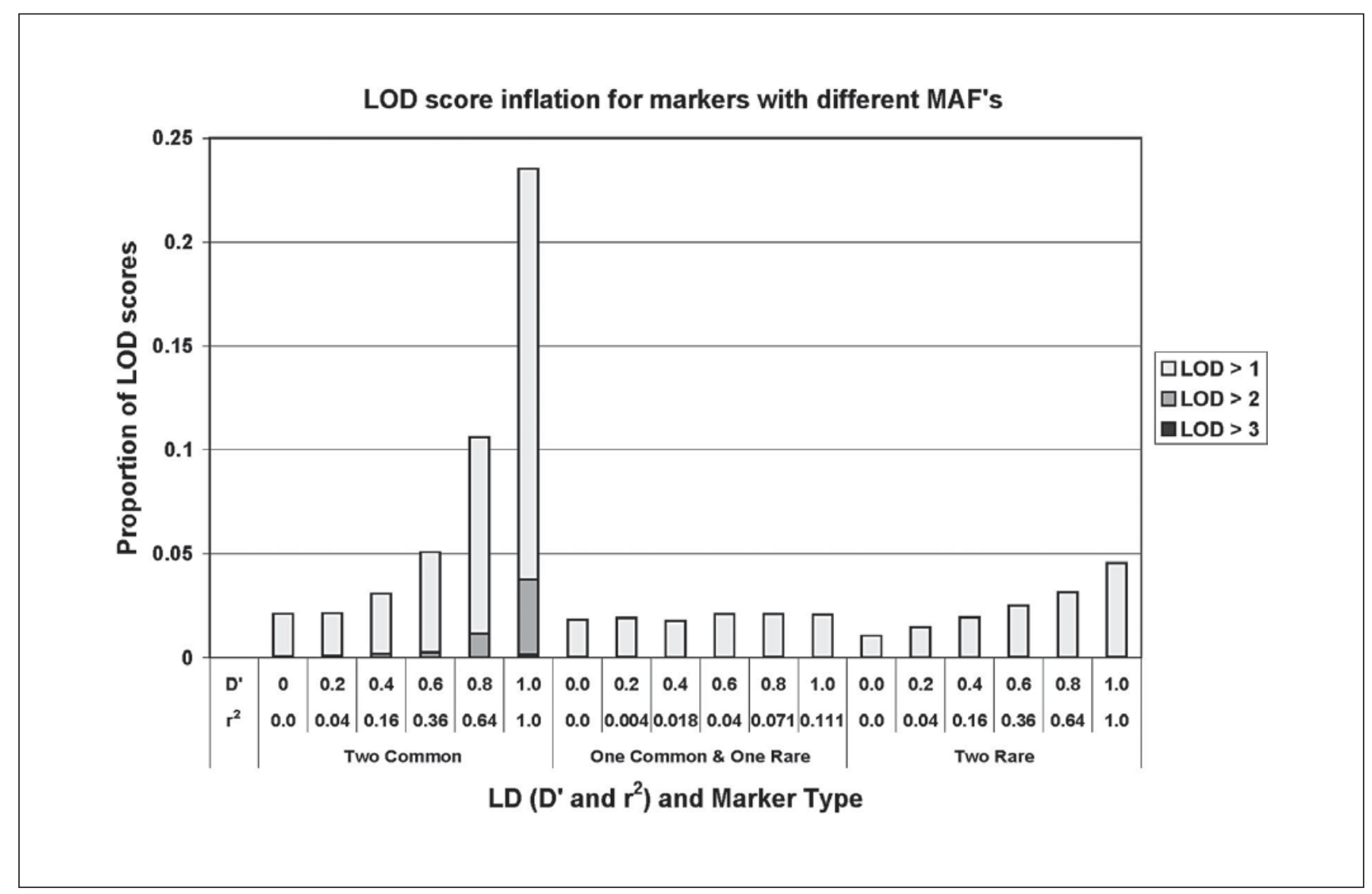

Fig. 4. The proportion of nonparametric multipoint LOD scores over 1,2 , and 3 for two common (MAF $=0.5)$, common and rare $(\mathrm{MAF}=0.1)$, and two rare allele frequencies over two markers in LD without parental genotypes only show inflation when $r^{2}$ levels go above 0.16 in the rare $\times$ rare case.

Inflation is not as great in the case with two rare allele markers compared to two common allele markers, but inflation is still noticeable at $\mathrm{D}^{\prime}$ levels as low as 0.4 (equivalent to an $r^{2}$ of 0.16 ). The one common with one rare marker scenario never reaches an $\mathrm{r}^{2}$ level of 0.16 and no inflation is observed. In this case $2 \%$ of the replicates produced a LOD score over 1, compared to two rare allele markers with $1.1 \%$ when $\mathrm{D}^{\prime}=0$ and $4.6 \%$ when $\mathrm{D}^{\prime}=1$.

Fig. 3. Nonparametric multipoint analysis without parents for two or four markers in LD are shown. The proportion of LOD scores greater than 1,2, and 3 are reported as $\mathrm{D}^{\prime}$ levels vary between 0 and 1 and as 2, 4, and 6 flanking markers in equilibrium are added to the multipoint. Inflation increases when markers in LD are added and decreases but is not eliminated as flanking markers in equilibrium are incorporated.

\section{Discussion}

Inter-marker LD dramatically increased the false positive rate of multipoint linkage analysis in simulated datasets when parental genotypes were missing. Linkage disequilibrium unaccounted for in linkage analysis, even as low as $\mathrm{D}^{\prime}=0.4$ across all of the markers, can still increase the likelihood of a spurious positive finding. Incorporating flanking markers not in LD tempered this effect, but the false positive rate was still higher than expected. Even higher levels of inflation were seen when four markers were in LD for nonparametric multipoint analysis. As expected no effect of inter-marker LD was observed when parental genotypes were available.

Two popular measures of LD, $\mathrm{D}^{\prime}$ and $\mathrm{r}^{2}$, are not equivalent when the markers' allele frequencies are different; further complicating evaluation of the impact of LD on 
linkage analysis. $\mathrm{D}^{\prime}$ and $\mathrm{r}^{2}$ both maximize to 1 only when the markers in LD have equal allele frequencies. When two markers have disparate allele frequencies and the maximum value of $\mathrm{LD}$ may achieve the maximum of $\mathrm{D}^{\prime}$ of 1 , however $r^{2}$ will be lower, often dramatically lower exhibiting less sensitivity to allele frequency misspecification. Our simulation studies show that whether allele frequencies are rare or common, LOD scores only showed inflation when the $\mathrm{r}^{2}$ measure was above 0.16 . Our results indicate that the $\mathrm{r}^{2}$ measure should be used when addressing the possibility of inflated multipoint LOD scores due to inter-marker LD.

Inter-marker LD did not affect two-point LOD scores because individual marker allele frequencies were correctly specified in Hardy-Weinberg equilibrium while haplotype frequencies were not, violating the assumption of linkage equilibrium. Large discrepancies between the two-point and multipoint results in a small region may be an indication that the assumptions of the multipoint map are not correct. In these simulated data, unaccounted for LD is inflating the multipoint LOD scores. In this case, two-point LOD scores may be more reliable than multipoint scores. Multipoint mapping helps localize the gene and can increase the genetic informativeness, but as reiterated here, it is more vulnerable to spurious results when parameters are misspecified.

Most analysis programs assume linkage equilibrium when inferring parental haplotype frequencies and such estimates may be quite different from the true haplotype frequencies (table 2). Determining how to evaluate and best incorporate LD into linkage analysis is a topic under intense debate, in large part due to the tremendous resources available through the HapMap initiative [9]. The simplest solution to combat the effect of regions of strong LD is to re-code haplotype blocks as a single multiallelic marker with the correct haplotype frequencies as allele frequencies. Analysis of the re-coded data would be straightforward and not prone to inflation, however the logistics of delineating these blocks and properly coding them can be difficult and time-consuming. Analyzing only haplotype tagging SNPs may be useful here.

Terwilliger and Ott [10] proposed an extension of the Haplotype Relative Risk method to estimate the extent of LD and increase the power of the study. Another promising solution that directly addresses this issue is included in Merlin version 1.0, where a clustering strategy for markers in tight LD corrects for the inflation in LOD scores [11]. Incorporating LD into analytical methods can only be done when its presence has already been noted, but unaccounted for LD will still affect the results and create problems correctly estimating parental haplotype frequencies.

The temptation to select markers that produce the highest LOD scores is great, particularly in diseases where samples from multiple generations are rare, limiting the power of proposed studies. Despite the potential to increase informativeness and improve localization, multipoint LOD scores should be viewed with caution when there are missing parental genotypes and the multipoint LOD score is much greater than the two point LOD scores. Linkage disequilibrium must be evaluated between SNPs and even close microsatellite markers, because low levels can still create inflated LOD scores purely due to marker-marker LD rather than disease-marker LD. The development of statistical methods that accurately account for LD in multipoint analysis will help to capitalize on all the available information resulting from the current emphasis on linkage disequilibrium and haplotype mapping.

\section{Acknowledgments}

Special thanks to Evadnie Rampersaud and Kristen Bastress for their support on this project, as well as the anonymous reviewers of this paper who provided very helpful comments that strengthened our conclusions. We gratefully acknowledge support from these grants: NS39818, ES11961, ES11375, MH59528, AG19757, AG021547, EY015216, HD39948, HL073389, and NS26630. 


\section{References}

$\checkmark 1$ Matise TC, Sachidanandam R, Clark AG, Kruglyak L, Wijsman E, Kakol J, Buyske S, Chui B, Cohen P, de Toma C, Ehm M, Glanowski S, He C, Heil J, Markianos K, McMullen I, Pericak-Vance MA, Silbergleit A, Stein L, Wagner M, Wilson AF, Winick JD, WinnDeen ES, Yamashiro CT, Cann HM, Lai E, Holden AL: A 3.9-centimorgan-resolution human single-nucleotide polymorphism linkage map and screening set. Am J Hum Genet 2003; 73:271-284.

2 Cardon LR, Abecasis GR: Using haplotype blocks to map human complex trait loci. Trends Genet 2003;19:135-140.
-3 Clerget-Darpoux F, Bonaiti-Pellie C, Hochez $\mathrm{J}$ : Effects of misspecifying genetic parameters in lod score analysis. Biometrics 1986;42:393399.

4 Ott J: Linkage analysis with misclassification at one locus. Clin Genet 1977;12:119-124.

$\checkmark 5$ Risch N, Giuffra L: Model misspecification and multipoint linkage analysis. Hum Hered 1992;42:77-92.

6 Huang Q, Shete S, Amos CI: Ignoring linkage disequilibrium among tightly linked markers induces false-positive evidence of linkage for affected sib pair analysis. Am J Hum Genet 2004; 75:1106-1112.

$\checkmark 7$ Bass MP, Martin ER, Hauser ER: Pedigree generation for analysis of genetic linkage and association. Pac Symp Biocomput 2004:93103.
-8 Hauser ER, Boehnke M: Genetic linkage analysis of complex genetic traits by using affected sibling pairs. Biometrics 1998;54:1238-1246.

9 Gibbs RA, The International HapMap Consortium: The International HapMap Project. Nature 2003;426:789-796.

10 Terwilliger JD, Ott J: A haplotype-based 'haplotype relative risk' approach to detecting allelic associations. Hum Hered 1992; 42:337346.

11 Abecasis GR, Wigginton JE: Linkage analysis with markers that are in linkage disequilibrium. Annual Meeting of the American Society of Human Genetics 2004, Abstract 94. 